\title{
Efecto de dos modelos de la línea de tres puntos sobre variables relacionadas con la acción de juego en minibasket femenino Effect of two models of three points line in female mini-basket
}

\author{
*José L. Arias Estero, *Francisco M. Argudo Iturriaga, ${ }^{* *}$ José I. Alonso Roque \\ * Universidad Autónoma de Madrid (España), **Universidad de Murcia (España)
}

\begin{abstract}
Resumen: El objetivo del estudio fue comparar la línea de tres puntos delimitada por el área de la zona restringida y la línea de tres puntos delimitada por un área rectangular, para examinar cuál de los dos diseños de la línea puede contribuir a favorecer en mayor medida el desarrollo de las habilidades de los jugadores de minibasket. En un primer campeonato se jugó con la línea de tres puntos demarcada por la zona restringida (1642 posesiones de balón) y en un segundo con una línea de tres puntos rectangular (1669 posesiones de balón). Se utilizó la metodología observacional, mediante un diseño tipo seguimiento, idiográfico, multidimensional. Tras formar a cuatro observadores, la fiabilidad del registro obtenida a través del coeficiente de concordancia Kappa, alcanzó valores entre .90 y .98. La relación entre cada campeonato y todos los criterios fue significativa $(P=.000)$. Al jugar con la línea de tres puntos delimitada por la zona restringida se observan mayores porcentajes con respecto a la delimitada por el área rectangular, en relación a las posesiones de balón desarrolladas mediante transición $(60.1 \%$ vs. $51.5 \% ; Z=-3.7, P=.000)$ y el número de lanzamientos $(62.9 \%$ vs. $44.9 \% ; Z=-2.65$,
\end{abstract} $P=.007)$.

Palabras clave: Baloncesto, minibasket, modificación de reglas, línea de tres puntos.

Abstract: The aim of this study was to examine the differences in game dynamics by comparing two disparate ways of conceiving the three-point line in mini-basketball into a competitive, real context. During the first championship, a three-point line delimited by the restricted zone (1.642 ball possessions) was played while during the second one, a rectangular three-point line (1.669 ball possessions). The observational methodology was used through a follow-up, ideographic, multidimensional design type. After training four observers, the register's reliability, which was obtained by the Kappa concordance coefficient, achieved values between 0.90 and 0.98 . The relation between each championship and all the criteria was significant $(P=$ 0.000). In playing with the three-point line delimited by the restricted zone, greater percentages were observed than with the three-point line delimited by the rectangular area with regard to the ball possessions developed through transition $(60.1 \%$ vs. $51.5 \% ; Z=-3.7, P=0.000)$ and to the shooting number $(62.9 \%$ vs. $44.9 \% ; Z=-2.65, P=0.007)$.

Key words: Basketball, mini-basketball, rule modification, three-point line.

\section{Introduction}

El Minibasket fue creado por Jay Archer, como una adaptación del baloncesto a las características y necesidades de los niños, con el objetivo de que éstos pudieran practicar y disfrutar de acuerdo con sus posibilidades (Asín, 1969; Daiuto, 1988; Tous, 1999). No obstante y de acuerdo con Piñar(2005), Piñar,Alarcón, Vegas, Carreño \& Rodríguez (2002) y Piñar et al. (2003), el lanzamiento realizado desde distancias superiores a cuatro metros y posiciones exteriores a la zona restringida es poco practicado en minibasket. Además, se ejecutan pocos lanzamientos de tres puntos, de los cuales pocos son encestados. Lo cual supone una limitación en el desarrollo de las habilidades del niño que practica este deporte. La Federación Española de Baloncesto(FEB) propuso una línea de tres puntos, delimitada por un área con forma rectangular a modo de área de fútbol, cuyo objetivo principal es paliar las limitaciones en cuanto al lanzamiento exterior. Ante esta regla los organismos responsables en cada comunidad autónoma han optado por adoptar esta línea de tres puntos o por utilizar la línea que delimita el área de la zona restringida como línea de tres puntos. Sin embargo, no se han realizado estudios que permitan determinar cómo influye la modalidad de la línea de tres puntos sobre las acciones de juego.

En este sentido, el análisis del marco situacional permite determinar el conjunto de comportamientos motores presentes en cada posesión de balón (Argudo, 2005). De manera, que el desarrollo de un marco situacional en transición favorece la práctica de más contenidos de juego y más variados. Además si el marco situacional se desarrolla en superioridad numérica se favorece la consecución de canastas. Para conocer si durante el partido las posesiones de balón finalizan con lanzamiento a canasta o de otra manera es conveniente estudiar la forma

\footnotetext{
Fecha recepción: 11-02-09 - Fecha envío revisores: 11-02-09 - Fecha de aceptación: 17-05-09 Correspondencia: Franciso Argudo Iturriaga

Departamento de Educación F́́sica Depote y Motricidad Human.

Ciudad Universitaria de Cantoblanco. 28049, Madrid

E-mail: quico.argudo@uam.es
}

de finalizar las posesiones. En minibasket el mayor número posible de posesiones de balón deberían terminar en lanzamiento a canasta por su importancia (De Rose, 2004; Karipidis, Fontinakis, Taxildaris \& Fatouros, 2001; Piñar, 2005), ya que esto se relaciona en minibasket con un aumento en la motivación de los jugadores (Chase, Ewing, Lirgg \& George, 1994; Vollmer \& Bourret, 2000). Y como consecuencia con un aumento en la cantidad de práctica y el desarrollo de esta habilidad de juego (Baker, Côté \& Abernethy, 2003). El lanzamiento a canasta es una de las habilidades motrices más significativas que el jugador de minibasket debe desarrollar (Maxwell, 2006; Piñar, 2005). Sin embargo, para que el jugador pueda desarrollar esta habilidad en condiciones óptimas, los tipos de lanzamientos deben ser variados en cuanto a su ejecución (Boyce, Coker \& Bunker, 2006; Maxwell, 2006). Por todo esto, sería ideal que en minibasket las posesiones de balón se desarrollasen en mayor medida mediante un marco situacional de transición y superioridad numérica, y que se aumentasen el número de posesiones que finalizasen en lanzamiento. De forma que estos lanzamientos fuesen variados en cuanto al tipo.

Las reglas especifican la naturaleza del problema del deporte y determinan las restricciones para su resolución (Gréhaigne, Godbout \& Bouthier, 2001). Son las que establecen las demandas energéticas (Platanou \& Geladas, 2006; Rampinini et al., 2007), las condiciones de juego (Hammond \& Hosking, 2005; Hammond, Hosking \& Hole, 1999) y las condiciones de los jugadores (Ackland, Schreiner \& Kerr, 1997; Carter, Ackland, Kerr \& Stapff, 2005).

Conscientes de las limitaciones de algunos reglamentos de juego, diferentes autores se han preocupado por su estudio, modificación y posterior análisis. Esto se ha hecho en edades adultas (Hammond, et al., 1999; Hughes, 1995) y en las primeras etapas de desarrollo de los individuos (Macpherson, Rothman \& Howard, 2006; Martens, Rivkin $\&$ Bump, 1984). En edades de iniciación en baloncesto, se investigó el tamaño del balón y la altura de la canasta: a) desde un punto de vista biomecánico (Regimbal, Deller \& Plimpton, 1992; Satern, Messier \& Keller-McNulty, 1989), b) en cuanto al ámbito de manejo del balón (Burton \& Welch, 1990) y c) desde una perspectiva de autoeficacia 
(Chase, et al., 1994). En minibasket, solo se ha encontrado el estudio realizado por Piñar (2005). En éste se modifican una serie de reglas, entre las que se encuentra la inclusión de una línea de tres puntos a cuatro metros del aro. Los resultados muestran un aumento del $17 \%$ en el rendimiento, un $13.7 \%$ más de posesiones de balón en las que se lanza desde el exterior de la zona restringida y un $9.6 \%$ más de posesiones en las que se lanza desde una distancia superior a cuatro metros.

No se han encontrado estudios que se preocupen por adecuar la línea de tres puntos, a las características de los jugadores de minibasket, para favorecer el lanzamiento y obtener éxito desde posiciones exteriores. Y puesto que las investigaciones más relevantes indican la importancia de la utilización de la línea de tres puntos desde los primeros estadios, se fijó como objetivo de este trabajo comparar la línea de tres puntos delimitada por el área de la zona restringida y la línea de tres puntos delimitada por un área rectangular. Esto se hizo para examinar cuál de los dos diseños de la línea de tres puntos contribuía a favorecer las acciones de juego que se vinculan con el desarrollo positivo de las habilidades de los jugadores de minibasket, en términos del marco situacional, la forma de finalizar la posesión de balón y el tipo de lanzamiento.

\section{Método}

\subsection{Participantes y muestra}

Participaron 67 jugadoras (edad 10.39, $\mathrm{s}=0.67$ años) de seis equipos de minibasket, que jugaron en la competición oficial durante la temporada 2006/2007. Las jugadoras llevaban practicando de forma reglada una media de 2.11, D.E. $=0.77$ años. La media de días de práctica era de 3.5, D.E. $=0.55$. Y el número de horas semanales de práctica era de media 5.33 , D.E. $=1.35$. Las jugadoras participaron en dos campeonatos, entre los que transcurrieron 30 días. Se analizaron 1642 posesiones de balón en el primer campeonato y 1669 en el segundo, centrando la atención sobre el jugador con balón. La muestra se seleccionó mediante un muestreo total de las acciones ocurridas (Anguera, 2003). Se fijaron dos criterios de inclusión: a) que en los dos campeonatos se debía jugar con las mismas jugadoras y b) que en el tiempo que transcurriera desde el primer campeonato hasta el segundo los entrenadores debían seguir entrenando de forma habitual, pero con la inclusión en la pista de la línea de tres puntos propuesta por la FEB. Antes de realizar la parte empírica del trabajo se recogieron los consentimientos informados de los padres $\mathrm{y}$ de los responsables de los equipos.

\subsection{Diseño}

Atendiendo al objetivo del estudio, se utilizó la metodología observacional, aceptada como metodología empírica para las ciencias del comportamiento (Anguera \& Blanco, 2003), mediante un diseño tipo seguimiento, idiográfico, multidimensional (Anguera, 2003), para analizar las dos campeonatos objeto de estudio: a) el primer campeonato fue cuando se utilizó la línea de tres puntos delimitada por el área de la zona restringida (ver Figura 1) y b) el segundo campeonato fue cuando se utilizó la línea de tres puntos delimitada por un área rectangular propuesto por la FEB (ver Figura 2).

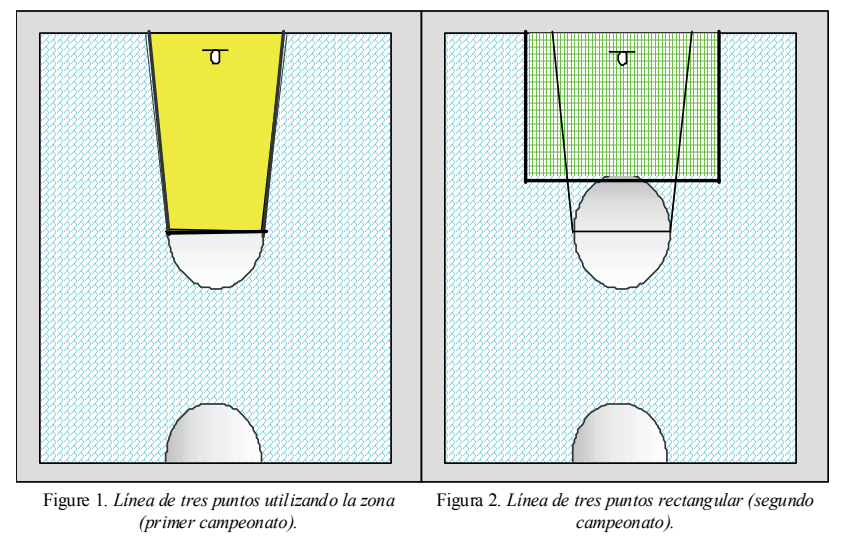

En ambos campeonatos se compararon los siguientes criterios:

1. Marco situacional en el que se efectuó cada posesión del balón: se registró el contexto en el que se desarrolló cada posesión del balón, atendiendo al número de jugadores y a la forma de desarrollar la posesión del balón. Se establecieron las siguientes categorías: 1) posicional en pista delantera de igualdad numérica, 2) posicional en pista delantera de superioridad numérica, 3) posicional en pista delantera de inferioridad numérica, 4) posicional en pista trasera de igualdad numérica, 5) posicional en pista trasera de superioridad numérica, 6) posicional en pista trasera de inferioridad numérica, 7) en transición en igualdad numérica, 8) en transición en superioridad numérica y 9) en transición en inferioridad numérica.

2. Forma de finalización de la posesión del balón: se registró la acción con la que se dejaba de tener el balón controlado. Se establecieron las siguientes categorías: 1) lanzamiento, 2) lanzamiento que no toca el aro y no se consigue encestar, 3) violación de los atacantes, 4) violación de los defensores, 5) robo del balón, 6) interceptación del balón, 7) salto entre dos, 8) error propio, 9) falta personal recibida y 10) decisión arbitral.

3. Tipo de lanzamiento: se registró la técnica de lanzamiento en cada posesión del balón. Se establecieron las siguientes categorías: 1) estándar sin salto, 2) estándar con salto, 3) carrera estándar, 4) carrera con bandeja, 5) carrera con gancho y 6) gancho.

\subsection{Procedimiento}

Como instrumento de observación se utilizó un sistema de categorías (Anguera \& Blanco, 2003). El instrumento de registro se construyó a partir de la adaptación de una hoja de cálculo del programa informático Microsoft Excel 2003, al que se le añadió una herramienta para capturar los vídeos y procesarlos (Virtual Dub, v. 1.7.0., Avery Lee).

Durante el primer campeonato se grabaron nueve partidos con dos cámaras de vídeo (JVC, Everio Full HD-GZ-HD7) colocadas transversalmente cada una en un campo de juego. En este campeonato se jugó con la línea de tres puntos delimitada por la zona restringida. Previo a la grabación del segundo campeonato, se delimitó con un rollo de $100 \mathrm{~m}$. de cinta adhesiva la línea de tres puntos propuesta por la FEB. Durante el segundo campeonato se grabaron otros nueve partidos siguiendo el mismo procedimiento. En ambos campeonatos se cumplieron los siguientes requisitos de constancia intersesional: a) las jugadoras participantes fueron las mismas, b) se jugaron todos los partidos en el mismo pabellón y dentro de este en dos pistas idénticas (28 x 15 m.), c) los partidos siguieron el mismo horario, d) el tiempo de descanso entre partidos fue de diez minutos, e) en cada partido se jugaron seis periodos de ocho minutos cada uno, f) hubo cuatro árbitros, g) el tamaño del balón fue el número cinco y h) la altura de las canastas fue de $2.60 \mathrm{~m}$.

Se elaboró un manual de formación que guió el proceso de entrenamiento y adiestramiento de los observadores. Fruto de este proceso y a partir de los trabajos de referencia se construyó el manual de instrucciones para los observadores. Este manual estaba formado por la explicación de: el sistema de categorías, la codificación de las categorías y el instrumento de registro.

Posteriormente se formó a cuatro observadores siguiendo las fases sugeridas por Anguera (2003), durante más de 40 horas. Finalizado el proceso de formación se evaluó el rendimiento a través de la fiabilidad en relación a un observador experto. Para el cálculo de la fiabilidad se utilizó el coeficiente de concordancia, mediante el estadístico Kappa. La fiabilidad alcanzó valores comprendidos entre .90 y .98. El último paso consistió en la toma de datos mediante un registro sistemático. La observación fue: activa, no participante, directa y de conductas espaciales (Anguera \& Blanco, 2003).

\section{4 . Análisis estadístico}

Los datos se recogieron mediante el instrumento de registro, para posteriormente capturarlos y archivarlos a través de un paquete estadístico (SPSS, v. 13.0. para Windows). A través de la prueba U de Mann-Whitney, se pudo analizar si existían diferencias significativas 
entre las medias de los criterios comparados, en función de los dos campeonatos objeto de estudio. Para conocer las correlaciones entre los diferentes criterios a analizar y los dos campeonatos objeto de estudio, se utilizó el test de Chi-Cuadrado. Mediante esta prueba se obtuvieron los valores de frecuencia, los porcentajes de cada campeonato y los residuos tipificados corregidos. El nivel de significación para las pruebas fue $P<.05$.

\section{Resultados}

3.1. Marco situacional en el que se desarrolla cada posesión de balón

Se encontraron diferencias significativas entre los dos campeonatos $(Z=-3.7, P=.000)$. El primercampeonato se relacionó significativamente con las posesiones de balón en transición (60.1\%) frente a las que se dieron en el segundo campeonato (51.5\%). En el segundo campeonato destacó el número de posesiones de balón que se desarrollaron de forma posicional $(48.5 \%)$, tanto en pista delantera como trasera, con respecto al $39.9 \%$ del primer campeonato $\left(\div^{2}=45.29\right.$, g.l. $\left.=2, P=.000\right)$ (ver Tabla 1).

\begin{tabular}{|c|c|c|c|c|c|}
\hline \multicolumn{6}{|c|}{ Tabla 1. Resultados del marco situacional. } \\
\hline & & \multicolumn{4}{|c|}{ Situación } \\
\hline & & \multicolumn{2}{|c|}{ Situación 1: zona restringida } & \multicolumn{2}{|c|}{ Situación 2: área rectangular } \\
\hline & & Recuento & $\%$ & Recuento & $\%$ \\
\hline \multirow{9}{*}{$\begin{array}{c}\text { Marco } \\
\text { Situacional }\end{array}$} & PDIg & 270 & 16.4 & 323 & 19.4 \\
\hline & PDS & 207 & 12.6 & 199 & 11.9 \\
\hline & PDI & 78 & 4.8 & 85 & 5.1 \\
\hline & PTIg & 48 & 2.9 & 134 & 8.0 \\
\hline & PTS & 19 & 1.2 & 26 & 1.6 \\
\hline & PTI & 33 & 2.0 & 42 & 2.5 \\
\hline & $\operatorname{TIg}$ & 466 & 28.4 & 442 & 26.5 \\
\hline & TS & 379 & 23.1 & 319 & 19.1 \\
\hline & $\mathrm{TI}$ & 142 & 8.6 & 99 & 5.9 \\
\hline \multicolumn{6}{|c|}{$\begin{array}{l}\text { PDIg: Posicional, pista Delantera, Igualdad numérica; PDS: Posicional, pista Delantera, Superioridad } \\
\text { numérica; PDI: Posicional, pista Delantera, Inferioridad numérica; PTIg: Posicional, pista Trasera, } \\
\text { Igualdad numérica; PTS: Posicional, pista Trasera, Superioridad numérica; PTI: Posicional, pista Trasera, } \\
\text { Inferioridad numérica; TIg: Transición, Igualdad numérica; TS: Transición, Superioridad numérica; TI: } \\
\text { Transición, Inferioridad numérica. }\end{array}$} \\
\hline
\end{tabular}

\subsection{Forma de finalización de la posesión de balón}

Las diferencias entre los dos campeonatos en cuanto a la forma de finalizar la posesión del balón fueron significativas $(Z=-2.65, P=.007)$. Se observó como en el primer campeonato el $62.9 \%$ de las posesiones finalizaron en lanzamiento, siendo el $44.9 \%$ lanzamientos que se encestaron o tocaron el aro. En el segundo campeonato el $56.3 \%$ de las posesiones de balón finalizaron en lanzamiento, ocurriendo los lanzamientos que se encestaron o tocaron el aro en un porcentaje del $43.2 \%$. Con respecto al resto de categorías, aquellas que se consideran negativas en posesión de balón, violación en ataque, robo e interceptación del balón y errores propios, sumaron el $25.4 \%$ en el primer campeonato y el $28 \%$ en el segundo campeonato $\left(\div^{2}=32.41\right.$, g.l. $\left.=9, P=.000\right)$ (ver Tabla 2).

\begin{tabular}{|c|c|c|c|c|c|}
\hline & & \multicolumn{4}{|c|}{ Situación } \\
\hline & & \multicolumn{2}{|c|}{ Situación 1: zona restringida } & \multicolumn{2}{|c|}{ Situación 2: área rectangula } \\
\hline & & Recuento & $\%$ & Recuento & $\%$ \\
\hline \multirow{10}{*}{$\begin{array}{c}\text { Forma de } \\
\text { Finalizar la la } \\
\text { Posesión }\end{array}$} & $\begin{array}{l}\text { Lanzamiento que se } \\
\text { Encesta / Toca el } \\
\text { Aro }\end{array}$ & 737 & 44.9 & 721 & 43.2 \\
\hline & $\begin{array}{l}\text { Lanzamiento no se } \\
\text { Encesta/ Toca Aro }\end{array}$ & 295 & 18.0 & 219 & 13.1 \\
\hline & $\begin{array}{l}\text { Violación de los } \\
\text { Atacantes }\end{array}$ & 94 & 5.7 & 112 & 6.7 \\
\hline & $\begin{array}{l}\text { Violación de los } \\
\text { Defensores }\end{array}$ & 0 & 0 & 1 & 0.1 \\
\hline & Robo de Balón & 94 & 5.7 & 108 & 6.5 \\
\hline & $\begin{array}{l}\text { Interceptación de } \\
\text { Balón }\end{array}$ & 67 & 4.1 & 89 & 5.3 \\
\hline & Salto entre Dos & 67 & 4.1 & 75 & 4.5 \\
\hline & Error Propio & 163 & 9.9 & 159 & 9.5 \\
\hline & $\begin{array}{l}\text { Falta Personal } \\
\text { Recibida }\end{array}$ & 107 & 6.5 & 145 & 8.7 \\
\hline & Decisión Arbitral & 18 & 1.1 & 40 & 2.4 \\
\hline
\end{tabular}

\subsection{Tipo de lanzamiento}

Se obtuvieron diferencias significativas entre los dos campeonatos $(Z=-3.4, P=.001)$. En el primer campeonato con respecto al segundo, se lanzó más de forma estándar con salto (37.2\% frente al $31.1 \%)$, en carrera con bandeja ( $2 \%$ frente al $0.8 \%$ ) y en gancho ( $1 \%$ frente al $0.2 \%)$ (ver Tabla3). La relación entre el primer campeonato y los lanzamientos estándar con salto, en carrera con bandeja y en gancho resultó directa $(\div 2$ $=37.14$, g.l. $=6, P=.000)$.

\begin{tabular}{|c|c|c|c|c|c|}
\hline \multicolumn{5}{|c|}{ Tabla 3. Resultados en relación al tipo de lanzamiento. } \\
\hline & & \multicolumn{4}{|c|}{ Situación } \\
\hline & & Situación 1: zona restringida & \multicolumn{2}{|c|}{ Situación 2: área rectangular } \\
\hline & Necuento & $\%$ & Recuento & $\%$ \\
\hline \multirow{7}{*}{$\begin{array}{c}\text { Tipo de } \\
\text { Agrumiento }\end{array}$} & $\begin{array}{c}\text { Estándar } \\
\text { Sin Salto }\end{array}$ & 54 & 37.1 & 728 & 43.6 \\
\cline { 2 - 7 } & $\begin{array}{c}\text { Estándar } \\
\text { Con Salto }\end{array}$ & 611 & 37.2 & 519 & 31.1 \\
\cline { 2 - 7 } & $\begin{array}{c}\text { Carrera } \\
\text { Estándar }\end{array}$ & 305 & 18.6 & 325 & 19.5 \\
\cline { 2 - 7 } & $\begin{array}{c}\text { Carrera con } \\
\text { Bandeja }\end{array}$ & 33 & 2.0 & 14 & 0.8 \\
\cline { 2 - 7 } & $\begin{array}{c}\text { Carrera con } \\
\text { Gancho }\end{array}$ & 12 & 0.7 & 15 & 0.9 \\
\cline { 2 - 7 } & Gancho & 17 & 1.0 & 3 & 0.2 \\
\hline
\end{tabular}

\section{Discusión}

El objetivo de este trabajo fue comparar la línea de tres puntos delimitada por el área de la zona restringida y la línea de tres puntos delimitada por un área rectangular. Esto se hizo para examinar cuál de los dos diseños de la línea de tres puntos contribuía a favorecer las acciones de juego que se vinculan con el desarrollo positivo de las habilidades de los jugadores de minibasket, en términos del marco situacional, la forma de finalizar la posesión de balón y el tipo de lanzamiento. En relación a este objetivo, se obtuvieron mayores porcentajes al jugar con la línea de tres puntos delimitada por el área de la zona restringida con respecto a la línea de tres puntos delimitada por un área rectangular en términos de posesiones de balón desarrolladas mediante transición, número de lanzamientos y variabilidad del lanzamiento. Esto mostró, de acuerdo con estudios previos (Piñar, 2005; Piñar, et al., 2002; Piñar et al., 2003), que las experiencias competitivas desarrolladas en el primer campeonato pueden favorecer en mayor medida el desarrollo de las habilidades de los jugadores de minibasket.

De acuerdo con Argudo (2005), el análisis del marco situacional permite determinar el conjunto de comportamientos motores presentes en cada posesión de balón. Atendiendo al número de jugadores implicados al finalizar las posesiones y a cómo fue el desarrollo de la posesión del balón, se encontraron diferencias significativas $(P=.000)$, a favor del primer campeonato en cuanto a las posesiones jugadas en superioridad numérica. Mientras que el segundo campeonato se relacionó con las posesiones jugadas en igualdad numérica. Igualmente, se encontraron diferencias significativas $(P=.000)$, en cuanto a las posesiones desarrolladas en transición a favor del primer campeonato. Por el contrario, el segundo campeonato se vinculó con las posesiones de balón posicionales. Estos datos mostraron como, al jugar con la línea de tres puntos delimitada por la zona restringida, las posesiones de balón respondieron en mayor porcentaje a una lógica de juego más natural en baloncesto, comenzando con la posesión del balón en pista trasera y evolucionando hasta la pista delantera. Aspecto éste positivo en las primeras edades, para favorecer la variabilidad en el aprendizaje de contenidos (Boyce, et al., 2006) de forma significativa (Green \& Magill, 1995; Williams \& Hodges, 2005). A la vez, este hecho posibilitó un juego más dinámico, que facilitó la consecución de canastas encestadas al no existir una estructuración clara de la defensa.

El lanzamiento a canasta debería ser la habilidad motora con la que se terminasen todas las posesiones de balón en minibasket (Piñar, 2005), por su importancia en el desarrollo de los niños (Karipidis, et al., 2001). En el primer campeonato se finalizó un $44.9 \%$ de las posesiones en 
lanzamiento que era encestado o tocaba el aro, en relación al $43.2 \%$ del segundo campeonato. El menor número de lanzamientos del segundo campeonato fue fruto de un mayor número de acciones negativas, parece ser, a causa de la inclusión de la línea de tres puntos con una mayor área geométrica. En cuanto al número de posesiones que finalizaron con lanzamiento, los resultados de este estudio fueron mayores que los encontrados por Piñar (2005) en minibasket femenino. Ésta encuentra que el $24.1 \%$ de las posesiones finalizan con lanzamiento en la modalidad $5 \times 5$ y el $29.1 \%$ en la modalidad $3 \times 3$. En este mismo estudio para el sexo masculino, se encuentra un valor del $24 \%$ para la modalidad $5 \times 5$ y del $27.1 \%$ para la modalidad $3 \times 3$. Los datos pusieron de manifiesto, que al jugar con la línea de tres puntos delimitada por la zona restringida se favoreció la ejecución de un mayor número de lanzamientos. Lo cual en minibasket es ideal, ya que a mayor número de lanzamientos se generan más opciones de encestar (Romanowich, Bourret \& Vollmer, 2007), y esto conlleva un aumento de la autoestima y de la motivación de los jugadores (Chase et al., 1994; Vollmer \& Bourret, 2000). Además, el desarrollo de las habilidades de las jugadoras, en este sentido, se pudo considerar más completo, en cuanto a mayores experiencias de enceste. Lo que contribuye al desarrollo del talento deportivo (Baker, et al., 2003; Baker,Horton, Robertson-Wilson \& Wall, 2003; Côté, Macdonald, Baker \& Abernethy, 2006).

Por su especial relevancia, como habilidad motriz específica con la que se concluyen el resto de habilidades y medios tácticos (Rojas, Cepero, Oña \&Gutiérrez, 2000), es necesario conocer el tipo de lanzamiento que utilizan los jugadores (Oudejans, Van de Langenberg \& Hutte, 2002; Tsitskaris, Theoharopoulos, Galanis \& Nikopoulou, 2002). En este sentido, en el primer campeonato, como en baloncesto (Rojas, et al., 2000), se utilizó más el lanzamiento estándar con salto. Por el contrario, en el segundo campeonato se utilizaron tipos de lanzamientos más estables, como es el caso del lanzamiento estándar sin salto y en carrera estándar. No obstante, parece que jugando con la línea de tres puntos delimitada por la zona restringida aumentó en mayor proporción la variedad de lanzamientos practicados. Este aspecto es muy importante en iniciación(Tsitskaris, et al., 2002), puesto que la variabilidad favorece el aprendizaje de un número importante de tipos de lanzamientos, lo cual, permite desarrollar un sistema cognitivo-motriz adaptable en este sentido (Schmidt, 1975).

Los resultados de este estudio muestran que las experiencias competitivas son diferentes, cuando se utiliza la línea de tres puntos delimitada por el área de la zona restringida, con respecto a la utilización de la línea de tres puntos delimitada por un área rectangular. Estas diferencias, al jugar con la línea de tres puntos delimitada por la zona restringida, posibilita que las jugadoras de minibasket: (a) realicen un mayornúmero de acciones de juego, porque se desarrollan más posesiones de balón bajo un marco situacional en transición; (b) finalicen un mayor número de posesiones de balón en lanzamiento; y (c) ejecuten lanzamientos más variados. A partir de este estudio son necesarios otros que analicen los resultados obtenidos sobre los criterios analizados y sobre otros. Además, futuros estudios deben intentar definir con precisión la distancia ideal a la que situar la línea de tres puntos en minibasket.

\section{Referencias bibliográficas}

Ackland, T. R., Schreiner, A. B. \& Kerr, D. A. (1997). Absolute size and proportionality characteristics of World Championship female basketball player. Journal of Sports Sciences, 15, 485-490.

Anguera, M. T. (2003). La observación. En C. Moreno Rosset (Ed.), Evaluación psicológica. Concepto, proceso y aplicación en las áreas del desarrollo y de la inteligencia (pp.271-308). Madrid: Sanz y Torres.

Anguera, M. T. \& Blanco, A. (2003). Registro y codificación en el comportamiento deportivo. En A. Hernández Mendo (Coord.), Psicología del Deporte (Vol. 2). Metodología (p. 6-34). Buenos Aires: Efdeportes (www.efdeportes.com).

Argudo, F. M. (2005). Conceptos, contenidos y evaluación táctica en waterpolo. Murcia: UCAM

Asín, G. (1969). Mini Basquet. Barcelona: Sintes.

Baker, J., Côté, J. \& Abernethy, B. (2003). Sport-specific practice and the development of expert decision-making in team ball sport. Journal of Applied Sport Psychology, $15,12-25$.
Baker, J., Horton, S., Robertson-Wilson, J., \& Wall, M. (2003). Nurturing sport expertise: factors influencing the development of elite athletes. Journal of Sports Science and Medicine, 2, 1-9.

Boyce, B. A., Coker, C. A. \& Bunker, L. K. (2006). Implications for variability of practice from pedagogy and motor learning perspectives: finding a common ground. Quest, $58,330-343$.

Burton, A. \& Welch, B. (1990). Dribbling performance in first-grade children: effect of ball and hand size and ball-size preferences. The Physical Educator, 3(1), 37-41.

Carter, J. E. L.,Ackland, T. R., Kerr, D.A. \& Stapff,A. B. (2005). Somatotype and size of elite female basketball players. Journal of Sports Sciences, 23(10), 1057-1063.

Chase, M.A., Ewing, M. E., Lirgg, C. D. \& George, T. R. (1994). The effects of equipment modification on children's self-efficacy and basketball shooting performance. Research Quarterly for Exercise and Sport, 65(2), 159-168.

Côté, J., Macdonald, D. J., Baker, J. \& Abernethy, B. (2006). When «where» is more important than «when»: birthplace and birthdate effects on the achievement of sporting expertise. Journal of Sports Sciences, 24(10), 1065-1073.

Daiuto, M. (1988). Basquetbol. Metodología de la enseñanza. Buenos Aires: Stadium.

De Rose, D. (2004). Statistical analysis of basketball performance indicators according to home/away games and winning and losing teams. Journal of Human Movement Studies, 47, 327-336.

Green, K. \& Magill, R. A. (1995). Variability of practice and contextual interference in motor skill learning. Journal of Motor Behavior, 27(4), 299-309.

Gréhaigne, J. F., Godbout, P. \& Bouthier, D. (2001). The teaching and learning of decision making in team sports. Quest, 53, 59-76.

Hammond, J. \& Hosking, D. (2005). Effectiveness of rule changes in netball. Communications to the 11th World Congress of Sport Psychology, Sydney, Australia.

Hammond, J., Hosking, D. \& Hole, C. (1999). An exploratory study of the effectiveness of rule changes in netball. Communications to the Fourth International Conference on Sport, Leisure and Ergonomics. Journal of Sports Sciences, 17, 916-917.

Hughes, M. (1995). Using notational analysis to create a more exciting scoring system for squash. In G Atkinson, \& T. Reilly (Eds.), Sport, Leisure and Ergonomics (pp. 243-247). London: E. \& F. N. Spon.

Karipidis, A., Fotinakis, P., Taxildaris, K. \& Fatouros, J. (2001). Factors characterizing a successful performance in basketball. Journal of Human Movement Studies, 41, 385-397.

Macpherson, A., Rothman, L. \& Howard, A. (2006). Body-checking rules and childhood injuries in ice jockey. Pediatrics, $117(2), 143-147$.

Martens, R., Rivkin, F. \& Bump, L.A. (1984). A field study of traditional and nontraditional children's baseball. Research Quarterly for Exercise and Sport, 55(4), 351-355.

Maxwell, T. (2006). A progressive decision options approach to coaching invasion games: basketball as an example. Journal of Physical Education New Zealand, 39(1), 58-71.

Oudejans, R. R.D., Van de Langenberg, R. W. \& Hutter, R. I. (2002). Aiming at a far target under different viewing conditions: visual control in basketball jump shooting. Human Movement Sciences, 21, 457-480.

Piñar, M. I. (2005). Incidencia del cambio de un conjunto de reglas de juego sobre algunas de las variables que determinan el proceso de formación de los jugadores de minibasket (9-11 años). Granada: Universidad de Granada.

Piñar, M. I., Alarcón, F., Palao, J. M., Vegas, A., Miranda, M. T. \& Cárdenas, D. (2003). Análisis del lanzamiento en el baloncesto de iniciación. En A. Oña \& A. Bilbao (Eds.), Libro de Actas del II Congreso Mundial de Ciencias de la Actividad Física y el Deporte. Deporte y Calidad de Vida (pp. 202-208). Granada: Editores.

Piñar, M. I., Alarcón, F., Vegas, A., Carreño, F. \& Rodríguez, D. (2002). Posiciones y distancias de lanzamiento durante la competición en minibasket. En A. Díaz, P. L. Rodríguez \& J. A. Moreno (Coords.), Actas del III Congreso Internacional de Educación Física e Interculturalidad. Murcia: Consejería de Educación y Cultura de la Región de Murcia.

Platanou, T. \& Geladas, N. (2006). The influence of game duration and playing position on intensity of exercise during match-play in elite water polo players. Journal of Sports Sciences, 24(11), 1173-1181.

Rampinini, E., Impellizzeri, F. M., Castagna, C., Abt, G., Chamari, K., Sassi, A., et al. (2007). Factors influencing physiological responses to small-sided soccer games. Journal of Sports Sciences, 25(6), 659-666.

Regimbal, C., Deller, J. \& Plimpton, C. (1992). Basketball size as related to children's preference, rated skill, and scoring. Perceptual and Motor Skills, 75, 867-872.

Rojas, F. J., Cepero, M., Oña, A. \& Gutiérrez, M. (2000). Kinematics adjustments in the basketball jump shot against an opponent. Ergonomics, 43, 1651-1660.

Romanowich, P., Bourret, J. \& Vollmer, T. R. (2007). Further analysis of the matching law to describe two- and three-point shot allocation by professional basketball players. Journal of Applied Behavior Analysis, 40(2), 311-315.

Satern, M. N., Messier, S. P. \& Keller-McNulty, S. (1989). The effects of ball size and basket height on the mechanics of the basketball free throw. Journal of Human Movement Studies, 16, 123-137.

Schmidt, R. A. (1975). A schema theory of discrete motor skill learning. Psychological Review, 82, 225-260.

Tous, J. (1999). Reglamento de baloncesto comentado. Barcelona: Paidotribo.

Tsitskaris, G., Theoharopoulos, A., Galanis, D. \& Nikopoulou, M. (2002). Types of shots used at the Greek national basketball championships according to the division and position of players. Journal of Human Movement Studies, 42, 43-52.

Vollmer, T. R. \& Bourret, J. (2000). An application of the matching law to evaluate the allocation of two- and three-point shots by college basketball players. Journal of Applied Behavior Analysis, 33(2), 137-150.

Williams, A. M. \& Hodges, N. J. (2005). Practice, instruction, and skill acquisition in soccer: challenging tradition. Journal of Sports Sciences, 23(6), 637-650. 